\title{
La expresión de la violencia instrumental y simbólica en Facebook y Twitter
}

\section{The expression of instrumental and symbolic violence on Facebook and Twitter}

\author{
Marco Fabio Barrera Márquez (D) Lidia Rodríguez Alfano \\ ${ }^{1}$ Universidad Autónoma de Nuevo León, Nuevo León, México. \\ fabiobarreravmx@gmail.com \\ ${ }^{2}$ Universidad Autónoma de Nuevo León, Nuevo León, México. \\ lidia.rodriguezal@uanl.edu.mx
}

GACCESO ABIERTO / OPEN ACCESS

Cita: Barrera Márquez, Marco Fabio, Rodríguez Alfano, Lidia (2020). La expresión de la violencia instrumental y simbólica en Facebook y Twitter. Textos en Proceso, 6(1), pp. 160-171. https://doi.org/10.17710/tep.2020.6. 1.9barrerarodriguez

Editores: Esperanza Alcaide Lara, Universidad de Sevilla, Ana Pano Alamán, Università di Bologna

Recibido: 26-02-2020

Aceptado: 25-05-2020

Conflicto de intereses: Los autores han declarado que no poseen conflicto de intereses.

Copyright: @ Marco Fabio Barrera Márquez, Lidia Rodríguez Alfano. Esta obra está bajo licencia Creative

\section{Resumen}

Con un enfoque multidisciplinario, se describen los procedimientos discursivos mediante los cuales se expresa la violencia instrumental (Fuentes y Alcaide, 2008) y simbólica (Bourdieu, 1990) en una muestra construida para realizar un estudio piloto dentro de una investigación más amplia. En el corpus seleccionado de Facebook y Twitter, los usuarios se identifican como miembros de dos grupos intersubjetivos (Bucholtz y Hall, 2005): "chairos", simpatizantes de la izquierda, y "derechairos", de ideas políticas conservadoras. La metodología es descriptiva apoyada en cuantificaciones.

Palabras clave: redes sociodigitales, procedimientos discursivos, grupos intersubjetivos, identidad interactiva, violencia instrumental y simbólica.

\begin{abstract}
With a multidisciplinary approach, the discursive procedures by which instrumental (Fuentes \& Alcaide, 2008) and symbolic violence (Bourdieu, 1990) are expressed are described in a sample constructed to carry out a pilot study within a broader investigation. In the selected corpus of Facebook and Twitter, users identify themselves as members of two intersubjective groups (Bucholtz and Hall, 2005): "chairos", sympathizers of the left, and "derechairos" of conservative political ideas. The methodology is descriptive supported by quantifications.
\end{abstract}

Keywords: sociodigital networks, discursive procedures, intersubjective groups, interactive identity, instrumental and symbolic violence. 


\section{Introducción}

El contenido de este artículo es parte de la investigación doctoral "La (re)construcción discursiva de la realidad en redes sociodigitales". Se reportan resultados del estudio piloto en cuya realización se persigue precisar la metodología que habrá de seguirse en el análisis de la muestra total y la interpretación de los resultados.

Desde una perspectiva multidisciplinaria que, a partir de los estudios del discurso, articula planteamientos de la filosofía y las ciencias sociales, describimos los procedimientos discursivos que se presentan en una muestra -construida ad hoc para el estudio piloto- de Facebook y Twitter. Estas plataformas de las redes sociodigitales son entendidas, con Winocur y Sánchez (2016), como herramientas que permiten al usuario ampliar sus recursos y tender redes de contactos horizontales en el mundo virtual.

Para la construcción del corpus, primeramente, hicimos un recorte de la muestra construida para la investigación doctoral, que se basa en el modelo propuesto por Salgado (2018, p. 125-128) para conformar corpus de redes sociodigitales que pudieran ser explorados desde coyunturas o eventos histórico. Bajo este criterio, seleccionamos el tema de la política mexicana como fue tratado en participaciones producidas en Facebook y Twitter durante el periodo de las elecciones para presidente de la república de 2018. Además, consideramos otros dos temas de índole política que no forman parte de la muestra de la tesis de doctorado, a fin de elegir aquellos que propician mayor interacción entre los usuarios. De acuerdo con Google Trend (2019) y Trendinalia (2019), estos temas eran: el desabasto de gasolina sufrido en México en enero y febrero de 2019 -como consecuencia de una estrategia gubernamental contra la corrupción-; y la aplicación de la "consulta ciudadana" sobre la construcción del nuevo aeropuerto de la Ciudad de México.

Para el recorte temporal, aprovechamos los datos arrojados por Google Trend (2019), según los cuales el periodo de junio de 2018 a febrero de 2019 fue el lapso en que se generó mayor participación de usuarios en la web sobre estos temas.

El corpus definitivo para este estudio quedó conformado por 150 emisiones, cuya distribución temática es: 50 participaciones sobre las elecciones, 50 acerca del desabasto de gasolina y 50 de la "consulta ciudadana". Del total, 67 son de Facebook y 83 de Twitter. debido a que esta segunda plataforma motiva una mayor participación de los usuarios en temas de política.

Enseguida examinamos el uso de procedimientos discursivos que se presentan en este corpus.

\section{Descripción y análisis de los procedimientos discursivos}

La pregunta general planteada en este estudio es: ¿cuáles procedimientos discursivos, presentes en el corpus seleccionado de Facebook y Twitter, manifiestan actitudes de discriminación y violencia? La respuesta se halla en los resultados de la ruta analítica que seguimos para evaluar el influjo bidireccional de las redes sociodigitales y el sujeto, y que comprende tres tipos de dinámicas del discurso que se inciden mutuamente y que, para fines del presente artículo, designamos: 'etiquetado e identificación', 'formación de grupos intersubjetivos', y 'violencia instrumental y simbólica'. Enseguida describimos estas tres dinámicas: 


\subsection{Etiquetado e identificación}

El procedimiento de etiquetado consiste en denigrar al otro con el fin de conseguir la aprobación de un punto de vista dado, por lo que, con base en propuestas de Fuentes y Alcaide (2008), lo entendemos como un tipo de estrategia verbal que puede catalogarse como "violencia instrumental".

$\mathrm{Su}$ análisis nos llevó a descubrir una relación muy estrecha entre las participaciones dentro del corpus estudiado y el contexto político donde se produjeron, ya que el usuario distingue los grupos de la derecha y la izquierda de la política mexicana. A partir de esta distinción, surge una dinámica discursiva que consiste en ofender al "oponente", adjudicándole sobrenombres de implicación negativa, esto es, mediante el empleo de las etiquetas (Lipovetsky, 2016a): chairos, aplicada a los simpatizantes de la izquierda, y derechairos a los de la de derecha, como se ilustra en los siguientes ejemplos:

1) TW @AquilesChavez |7 de ene de 2019: Con la novedad ... de que en Pachuca NO hay desabasto de Gasolina .... y lo mejor ... hasta bajo de precio ... lo yo .. que soy un ciudadano común y corriente!!!! Aclaró .... Y no ando de Chairo y/o Derechairo!!!!

2) TW @ Cab_Negro_90 | 6 de ene de 2019: Al derechista este le arde que AMLO sea presidente. Por un desabasto nada grave, en zonas muy específicas, haces un drama. Nadie ha dicho que no hay desabasto. Debes aprender a leer, don derechairo.

En ambos tuits, se presenta el procedimiento discursivo del etiquetado. En (1), el emisor se deslinda de recibir el apelativo de chairo y/o derechairo, mientras que en (2) se ataca a una persona que "le arde" el triunfo de Andrés Manuel Lopez Obrador (AMLO), por lo cual es catalogado como derechista o derechairo. El sujeto crea una identidad opuesta al otro, el chairo busca en el derechario (y viceversa) lo que no es él, para conformarse a sí mismo (Hall, 1996, p. 18); y, al mismo tiempo, construye la identidad del otro con rasgos y características negativas, asignándole una etiqueta.

Sobre las etiquetas o hashtag, Mancera Rueda y Pano Alamán (2013) identifican su presencia en Twitter marcada con el signo de \#. En el corpus estudiado aparece el uso de ese signo en las dos plataformas:

3) TW@MarioAja9|23 de enero de 2019: ¡Ah! ¿No qué no? ¿Dónde están todos los \#chairos estúpidos que me estaban diciendo que el \#desabasto era por el combate al \#Huachicoleo? ${ }^{1}$ Los leo...

4) TW@LtMcClane69 | 16 ene. 2019: Desabasto de gasolina no tendrá afectación mayor: Bank of America, quiubo \#derechairos, no es el fin del mundo, no el \#apocalipsis, ya ven... la cosa es calmada.

5) FB José Carlos López García | 18 de enero de 2019: Buenos días \#Chairos, ya se arrepintieron?????

\footnotetext{
${ }^{1}$ Se llamó "Huachicoleo" (palabra derivada del latín aquati, que significa aguado) a la práctica del robo de combustible de los ductos que lo conducían a su distribución en gasolinerías.
} 
6) FB Abraham Giron | 10 de enero del 2019: Así el \#EZNL² y muchos \#DereChairos 周 (코 \#MenteDerechaira.

Como puede observarse, la función del signo \# no se limita a marcar las etiquetas (chairos y derechairos) sino que, como señalan Mancera Rueda y Pano Alamán, su función es "ampliar la información sobre un tema y favorecer su extensión de forma viral" (2013, p. 77); y, por otra parte, en este estudio encontramos que las etiquetas pueden aparecer sin esa marca:

7) TW@ElChairoPriet | 18 de agosto de 2018: Disculpen, soy chairo, voté por AMLO y necesito una consulta popular sobre si puedo entrar a la sala VIP del Cinépolis o estoy condenado a ver la rosa de Guadalupe ${ }^{3}$ en harapos y descalzo afuera de un Elektra ${ }^{4}$.

8) FB Gabriel De la Sota 6 de noviembre de 2018: En EEUU se consulta. El derechairo muchas veces es ignorante.

Además, el etiquetado, con o sin marca, puede encontrarse en toda la web, desempeñando otras funciones, como el reconocimiento de la identidad y la formación de grupos intersubjetivos y/o su uso para violentar al otro.

La identificación por parte del sujeto, de sí mismo y del otro, es el procedimiento que, dentro de las redes puede darse, precisamente, por medio de una etiqueta (Mancera Rueda y Pano Alamán, 2013 y Lipovetsky, 2016a).

Según Lipovetsky, culturalmente los sujetos realizan un etiquetado donde se adjudican implicaciones culturales (2016b, p. 11). En el corpus examinado, vemos cómo el usuario realiza el etiquetado como una dinámica discursiva para construir la identidad del otro, en su mayoría, negativa.

\subsection{Formación de grupos intersubjetivos}

La formación de grupos intersubjetivos (Husserl, 1986 y Bucholtz y Hall, 2005) es un resultado del proceso de creación de la identidad interactiva (Yus, 2014 y Hall, 1996) que se realiza a través del uso de etiquetas.

Cuando el sujeto reconoce los grupos 'nosotros' y 'ellos', surge una dinámica discursiva mediante la cual un grupo rechaza por completo al otro. Este proceso discursivo no coincide con la propuesta de Husserl (1986) sobre la intersubjetividad, que este filósofo define como una identificación en la cual el sujeto se ve a través de los ojos del otro, lo cual se traduce en empatía intergrupal.

La intersubjetividad que encontramos en la muestra analizada sirve como medio para dar origen a "diversas relaciones superpuestas y complementarias, que incluyen, autenticidad/artificio y autoridad/ilegitimidad" (Bucholtz y Hall, 2005, p. 13). Este funcionamiento se evidencia en:

\footnotetext{
${ }^{2}$ Las siglas EZNL refieren al Ejército Zapatista de Liberación Nacional, grupo militar que se rebeló contra la presidencia de la república el 1 de enero de 1994 y hasta 2006 fue militarizado.

3 "La Rosa de Guadalupe" es el título de una telenovela muy popular en México.

4 "Elektra" es el nombre de una compañía de servicios financieros y comercio de electrodomésticos. En este tuit, el usuario crea una imagen donde se representa viendo la telenovela en uno de los aparadores donde está un aparato de TV encendido.
} 
9) TW@GarcesLaureano | 13 enero de 2019: A los chairos no les afecta el desabasto de gasolina porque no viajan en automóvil. Ellos viajan con resistol ${ }^{5}$.

10) FB Alexander García Villar | 10 ernero de 2019: Chairos: ¿Ya van a reconocer que se equivocaron con el Mesías, o necesitan mas muertos, mas despidos injustificados, más crisis por desabasto, más asesinatos en la calles, más corrupción en las dependencias? Despierten antes que sea demasiado tarde!

11) TW@Piromana696_| Febrero 2 de 2019: Ya hay desabasto de tamales. No tardan los derechairos en salir con:¡Es culpa de AMLO!

12) FB Ruben Celis $\mid 3$ de diciembre de 2018: Ay cabron, su máximo líder reconoce el desabasto...Pos no era paranoia de los derechairos...Tan bien peñetas neta..

En estos ejemplos se presenta el uso de las etiquetas para el ataque a los otros: en (9), se denigra al otro con sus acciones; en (10), el sujeto realiza un llamado a los chairos para que se den cuenta de que el "Mesías" que apoyan realiza acciones negativas; y en (11), el grupo de respaldo a AMLO ironiza las quejas de los derechairos por el desabasto. Si bien, los dos ejemplos anteriores presentan una relación de similitud/diferencia, (12) muestra un uso distinto de las etiquetas, que sirve al sujeto para identificarse a sí mismo de forma indirecta, volviendo ilegitima la opinión de los otros, a fin de obtener la autoridad para insultar.

Gracias a la relación de ilegitimidad $\rightarrow$ autoridad, surgen dinámicas que parten de presupuestos como: el otro no es igual a mí, en cuanto mi yo es un yo racional y crítico mientras el otro es ignorante, no merece el reconocimiento como similar.

Al respecto, coincidimos con Van Dijk, quien afirma lo siguiente: "como están representados los grupos sociales (Nosotros y Ellos) en las actitudes y los discursos (...) los que pertenecen al grupo están presentados positivamente, y los de otro grupo están presentados negativamente" (2006, p. 397).

No obstante, dado que la identidad del grupo se muestra como interactiva e intersubjetiva, el sujeto no se adherirá al grupo en forma permanente (al menos hasta el fin del intercambio comunicativo donde participa), entrará y saldrá de él según le convenga. El usuario utiliza al grupo como una máscara que le ayuda a proyectar el yo que quisiera ser (Goffman, 1997, p. 31).

En este sentido, los miembros del grupo se pueden identificar con la etiqueta si les conviene, pues el grupo, al ser intersubjetivo y dinámico, no obliga a una adhesión permanente. En consecuencia, la formación del grupo puede ser fuerte y a la vez efímera. El grupo brinda una identidad "estable" para el sujeto, pues se forma por medio de un discurso no expresado explícitamente, pero que, en lo implícito, subyace por medio de una serie de prejuicios y supuestos. Sin embargo, el grupo puede desacreditar la personalidad del sujeto y, entonces, el usuario entra en un juego en el que intenta evitar las disrupciones en su identidad (Goffman, 1997, p. 259).

\footnotetext{
${ }^{5}$ En México existen ciertos tipos de pegamentos que se utilizan como materias para drogarse, cabe señalar que por antonomasia estos adhesivos son reconocidos por el nombre de la marca más popular del país, la de Resistol.
} 


\subsection{Violencia instrumental y simbólica}

El procedimiento de la violencia verbal se ilustra en el siguiente ejemplo, donde aparece la etiqueta de derechairo empleada en forma peyorativa y dirigida al interior del grupo:

13) FB Diego Gonzalez | 2 de julio 2018: Tanto critican a los chairos que al final están peor que ellos. Ahí se ven, se quedan en su ridiculo mundo de fantasía y su fanatismo de derechairos.

Así, del ataque con sarcasmos hacia el grupo y la crítica a la persona que emitió su punto de vista, se pasa a la validación de las acciones que denuncia; $\mathrm{y}$, aunque el tema central era el desabasto de la gasolina, de inmediato el grupo responde con los siguientes comentarios:

14) FB Rafael Rafa $\mid 2$ de julio 2018: Ya se estan retirando los infiltrados.

15) FB Rubén Santiago $\mid 2$ de julio 2018: Chairos infiltrados se van solos.

16) FB Rocío Ardura $\mid 2$ de julio 2018: Adiós chairo cobarde... A donde vayas llegarán los reclamos de la joda que le pusieron al país!!!

El emisor del comentario (13) fue expulsado del grupo o salió del mismo debido a que su perfil se encuentra como invalidado o ya no tenía presencia dentro del grupo. Lo relevante es que, pese a que registró 15 reacciones positivas de personas que expresaban compartir su punto de vista, nadie se atrevió a defender su postura o a apoyarlo, quizá por el temor a sufrir la expulsión del grupo.

Podemos decir que el sujeto emplea estrategias de afiliación y autonomía en relación con la imagen social que desea proyectar (Bravo, 2010, p. 25), dado que se adhiere al grupo si este representa para él una imagen positiva, pero si muestra rasgos que considere negativos, buscará alejarse de él (como efectivamente lo hace el emisor del ejemplo 13).

Los usuarios que coinciden con (13) no buscan salir del grupo, pero tampoco arriesgan su imagen social emitiendo un comentario verbal, solo reaccionan de forma positiva. En este punto, cabe recordar que la identidad del sujeto en las redes está dada, entre otras, por una relación del tipo similitud/diferencia; por tanto, si bien es cierto que hay ciertas diferencias que el sujeto puede minimizar con el fin de adecuar la relación con el otro (Bucholtz y Hall, 2005, p. 13), mientras más discrepancias encuentre, más podrá ser catalogado como parte del grupo contrario. En (14), (15) y (16), se muestra el accionar de esta consecuencia, pues una vez que la opinión de (13) manifiesta muchas diferencias con el discurso de 'ellos', de inmediato se le otorga la etiqueta de 'chairo infiltrado'. A la vez, la defensa del grupo deja ver, en (14), (15) y (16), que de inmediato se utiliza la etiqueta verbal como forma de agresión al 'otro'.

Todos estos procedimientos discursivos muestran cómo los intereses del sujeto se anteponen a los del grupo. Esta es la estrategia de control y manipulación, si alguien no converge dentro de las ideas compartidas por el grupo, se le etiqueta negativamente y se le expulsa. Con todo, al tiempo que el sujeto etiquetado se convierte en 'otro', crea un contradiscurso para no ser parte de ese 'otro' al que ataca el grupo, sino un 'otro racional' que lo critica, y puede formar un nuevo grupo; pero, al mismo tiempo, se encuentra frente al 'otro' en circunstancias en las que solo 
sus expresiones y los prejuicios le ayudan a formar una visión de él (Goffman, 1997, pp. 265-266).

Sin duda la inmersión dentro de estos grupos intersubjetivos deja ver lo peligroso de estas dinámicas, pues el grupo puede justificar discursos de agresión a otro $\mathrm{y}$, al mismo tiempo, la fragilidad de la formación del grupo permite una dinámica de violencia verbal entre sus integrantes.

Como resultado preliminar del análisis del empleo de las etiquetas chairo y derechairo, concluimos lo siguiente:

A. Chairos y derechairo se oponen discursivamente.

B. Dentro del grupo de derecha se da una ruptura con sus dinámicas propias y, por tanto, con las que el "orden del discurso" (Foucault, 1987) ha vuelto no permisibles. De este grupo surge otro que se identifica con un discurso que añade, a la etiqueta de derechairo, la carga semántica del fanatismo.

C. El emisor de un comentario que, aun habiéndose adscrito a la derecha, utiliza la etiqueta derechairo con la acepción de fanatismo, es expulsado del grupo, pero sigue sin coincidir con las ideas de los grupos etiquetados como chairos. Al tiempo que utiliza la etiqueta derechairo, ataca a los chairos, y se defiende de los ataques de su antiguo grupo.

D. Como forma de ataque, los grupos de izquierda utilizan la etiqueta derechairo para desprestigiar al grupo de ideas conservadoras.

El proceso anterior crea distintas etiquetas y realiza diferentes modificaciones de un discurso. Los miembros de dos grupos se pueden oponer mutuamente en los discursos que emiten. No obstante, se pueden aliar a otros grupos con un discurso compartido. Quienes etiquetan a los de derecha como derechairos pueden comulgar con el discurso de éstos, sin embargo, no apoyan sus actividades y terminan por oponerse en ese sentido.

Cabe señalar que el objetivo de los sujetos no es la agresión hostil al otro. Más bien buscan que el otro, del mismo grupo o no, entienda y se una a su propio punto de vista. En consecuencia, la violencia que utilizan los sujetos dentro de las redes tiene una naturaleza instrumental (Fuentes y Alcaide, 2008, pp. 17-18) y simbólica (Bourdieu, 1990). Para apoyar esta afirmación, presentamos los siguientes ejemplos:

17) FB Armando Mayorga Dávila| 12 de enero de 2019: Entiendan pinches chairos pendejos, estamos a favor de México al mil por ciento, por eso protestamos por los 150 millones a la basura del aeropuerto, la Guardia Nacional y la falta de gasolina $\tan \tan \ldots$

18) FB Miguel Barba | 13 de diciembre de 2018: DERECHAIROS!!! DERECHAIROS!!! COMO SE LLENAN EL HOCICO DICIENDO QUE LOS MÁS PREPARADOS PARA GOBERNAR MÉXICO...AHI TIENEN A SUS TITULADOS,CON DOCTORADOS Y LICENCIATURAS,LOS MÀS PREPARADOS DE MÉXICO??? SU ORGULLO NO LES DEJA VER LA REALIDAD...CREO QUE ALGO LES HACE FALTA EN LA CABEZA PARA QUE VEAN MEJOR LAS COSAS.

19) TW@ChairoMexicano 29 de octubre de 2018: Tal parece que los derechairos tendrán como lema de "batalla" en redes el hashtag \#MeDuelesMéxico, lo usaron el día posterior a la elección donde AMLO arrasó con el 53\% de los votos y el día posterior a la consulta donde la opción de Santa Lucía arrasó con el 69\%. Desamparados. 
20) TW@plumadisidente | 5 de diciembre de 2018: AMLO recibió un "bastón del mando" étnico y sus chairos milagrosamente ya aman a los indígenas pero a mi, no se me olvida que en la consulta votaron a favor de un Tren que destruirá los ejidos mayas y a Marichuy la ofendieron sólo por ser de origen nahua.

En (17) y (18), el usuario expresa literalmente la búsqueda de un entendimiento: (17) comienza diciendo "Entiendan", y (18) afirma "SU ORGULLO NO LES DEJA VER LA REALIDAD". Ambos buscan que el otro entienda "la realidad". Lo mismo sucede con (19), quien incluso arroja cifras para evidenciar su punto; (20), por su parte, contrasta dos puntos de vista, con lo que pretende que otro se adhiera más fuertemente a su postura.

Al respecto, es importante destacar que ninguno de los usuarios deja de utilizar la violencia instrumental. En específico, emplean subestrategias de discriminación como el insulto, el poner en evidencia y, sobre todo, el acusar al otro de desconocer la realidad (Fuentes y Alcaide, 2008, p. 24).

En Twitter, Pano Alamán evidencia cómo la narrativa se integra por múltiples voces que se traducen en una polifonía. Afirma: "La continuación del relato obliga a todos los coautores a retomar, a modo de eco, los elementos esenciales del mismo" (2016, p. 186). La repetición de las estrategias de discriminación evidencia que la narrativa del grupo hace eco y reproduce de forma polifónica las estrategias de discriminación.

Según Salgado, dentro de Twitter se puede encontrar una autoconstrucción constante de sí y del otro (2017, p. 450). No obstante, esta discriminación, dado que se construye en la práctica y no solo dentro de las redes, también atraviesa la cultura que se alimenta de las redes sociodigitales, aunque puede presentar otras características.

\section{La violencia simbólica en las redes socio-digitales}

El proceso discursivo de etiquetado e identificación que conduce a la formación de grupos intersubjetivos produce una violencia simbólica (Bourdieu, 1990) que, de acuerdo con Byung-Chul Han (2016) se carga de positividad en cuanto, aparentemente, no busca el daño del sujeto a quien se dirige; más bien se ejerce mediante la emisión de un discurso que expresa mensajes positivos, de supuesta bondad, y de este modo se pretende legitimar la agresión intersubjetiva.

El peligro de la libertad en el uso de las redes es que, al hallarse el sujeto en un espacio donde supuestamente el poder se distribuye en forma más equitativa, su búsqueda de dominación se vuelve transparente o simbólica (Bourdieu, 1990, 72), por lo que se encuentra con un poder, según Foucault, horizontal (1980, p. 203); y, por tanto, le resulta factible "condensar" la mayor cantidad de poder para su causa.

La discriminación basada en la tradición moral plantea una violencia de bondades, una 'violencia positiva' (Byung Chul-Han, 2016, p. 8). Los comentarios que vierten los sujetos dentro de las plataformas virtuales tienen dos características inherentes: se asumen como poseedores de bondad y ejercen la discriminación del otro.

El sujeto se apropia de la posverdad para su beneficio (Haidar, 2018, p. 14), con lo que rompe completamente con la responsabilidad moral del discurso y entra en una racionalidad cínica (Sloterdijk, 2003, p. 22). Mientras la dinámica propicie un beneficio moral, económico o de prestigio para el sujeto, su interpretación será menos objetiva, y su empatía, menguada. 
Cada grupo persigue la meta de alcanzar la legitimación y el empoderamiento de su discurso (Haidar y Rodríguez, 1996, pp. 95-94). En las redes sociodigitales, el sujeto expulsa al otro, al ajeno a él, pues ante lo desconocido se esconde en sí mismo. En este sentido, las consecuencias coinciden con las que Byung Chul-Han expone acerca del sujeto en general:

Lo que lo enferma no es la retirada ni la prohibición, sino el exceso de comunicación y de consumo; no es la represión ni la negación, sino la permisividad y la afirmación. El signo patológico de los tiempos actuales no es la represión, es la depresión. La presión destructiva no viene del otro, proviene del interior $(2014$, p. 7$)$.

El problema de la agresión hacia el otro es que el sujeto se encuentra dentro de un espacio discursivo, dialógico e intersubjetivo; él mismo es el otro frente al otro. La creación de los discursos le impide comprobar un centro que lo respalde como la identidad que imagina en su experiencia. Por lo tanto, la violencia "positiva" también pierde su centro, se vuelve horizontal y ataca al sujeto por direcciones múltiples.

\section{A modo de conclusión}

1) Los sujetos tienen como principal objetivo conservar los privilegios que, según muestran, creen que les asegura la identidad que se han construido discusivamente. Al no conocer a su interlocutor, no saben qué privilegios le sume o reste a su visión de sí mismo. Este desconocimiento los lleva a la incertidumbre y, esporádicamente, al rechazo de quien no se acople a sus convicciones, lo que propicia la confrontación inter e intra grupal.

2) Si bien autores como Mancera Rueda y Pano Alamán (2013), así como las propias plataformas, explican el uso de la etiqueta o tag en Twitter, con una función de hipervínculo para la red social, precedido del símbolo numeral y con otras funciones discursivas específicas, en el corpus analizado se revela que el empleo de esta puede adquirir nuevas funciones en el discurso, al cambiar el enfoque de estudio, además de que se puede presentar sin la marca de numeral y en toda la web.

3) Dentro de los grupos, el etiquetado funciona como una dinámica de formación de identidades, pero también se usa poniendo sobrenombres con carga peyorativa a los sujetos, de modo que se constituye en un acto de discriminación que termina por propiciar la confrontación, así como violencia instrumental y simbólica entre los usuarios.

4) Con sustento en el análisis de la muestra seleccionada se puede afirmar que el funcionamiento del discurso en Facebook y Twitter pudiera revelar, en cierta medida, una ruptura (tal vez en ciernes) con los paradigmas discursivos rígidos que conducen a la institucionalización de discursos dominantes. El criterio en que se basa esta afirmación es que estas redes sociodigitales han abierto un espacio a la denuncia de las dinámicas que se debaten en los intercambios discursivos que difunden. En consecuencia, en el grado en que su difusión "evada" el control institucional, el conflicto creado en estas redes es libertario y promueve la autodisciplina en los discursos (Lipovetsky, 2016a) que se realiza en función de cada sujeto o sus relaciones.

5) En una referencia más amplia, con Byung Chul-Han, admitimos que, en Occidente, la ontología se relaciona con lo interior y la esencia; y, en consecuencia, se engendran identidades particularizadas, muchas veces 
impermeables entre sí y probablemente hostiles (2018, p. 40). El conflicto de las intersubjetividades, sumado a la creación de arquetipos a los cuales se puede suscribir el usuario de las redes sociodigitales, son condiciones adecuadas para emitir dinámicas verbales discriminatorias.

6) Los grupos intersubjetivos sirven para que el sujeto se construya una identidad grupal. Dado que este es el objetivo del usuario, en cuanto el grupo deje de precisar cualidades positivas, saldrá de él. Con ello, los grupos se vuelven muy variados, fugaces y frágiles.

7) La violencia dentro de las redes posee un carácter instrumental, cuyo objetivo es la discriminación del otro y al mismo tiempo, la búsqueda de poseer la verdad y la razón que le dé más valor a su yo frente al otro.

8) El insulto, el poner en evidencia y el acusar de desconocer la realidad son las estrategias de discriminación que utilizan los usuarios para violentar al otro.

9) El total de dinámicas encontradas en el corpus fue de 386, y en un $37 \%$ de ellas, se presentan las tres estrategias del inciso anterior. En el 74\% se utilizó sobre todo la de evidenciar y el acusar de desconocimiento, mientras que solo en $18 \%$ se recurrió al insulto.

10) Las estadísticas referidas en el punto anterior corroboran la importancia del "cómo se muestra" para el sujeto, pues el insulto puede hacer que el sujeto evoque un prejuicio negativo (Fuentes y Alcaide, 2008, p. 24); del mismo modo, el que abunde el uso de las estrategias de evidencia y de acusar deja en claro que lo que los usuarios quieren es ser reconocidos de forma positiva, a costas de la denigración del otro.

11) La etiquetas de Chairo y Derechairo fueron encontradas en el $72 \%$ de la muestra total, de ese porcentaje Facebook arrojó el 38\% y Twitter el 62\%, dejando ver que la segunda plataforma es la que posee una mayor participación política, pero apenas superior a la que presenta la plataforma de Facebook.

12) En cuanto a los temas, encontramos que el tópico del desabasto de gasolina generó más muestras, ya que presentó el $43 \%$ de las estrategias de discriminación; las elecciones presentaron el $31 \%$, y el tema de la consulta ciudadana, el $26 \%$.

13) Las estadísticas anteriores se las podemos atribuir también a la naturaleza de las redes, pues el tema más cercano temporalmente a nuestra investigación (el desabasto de gasolina) fue el que tuvo mayor número de participaciones; además, es el único de los tres que evidencia una problemática del actual gobierno, y por tanto del grupo de chairos.

14) En lo concerniente a las plataformas, en el total de comentarios de Facebook, el 79\% presentó dinámicas de violencia, mientras que, en el total de tuits, en Twitter, estas dinámicas aparecieron en el $89 \%$. Estos datos nos llevan a concluir que, si bien se trata de dos plataformas con dinámicas y procesos de interacción distintos, el problema de la discriminación y la violencia se extiende hacia dentro y fuera del ciberespacio.

\section{Referencias}

1. Bourdieu, P. (1990). Intelectuales, politica y poder. Buenos Aires: Eudeba UBA

2. Bravo, D. (2010). Pragmática socio-cultural. La configuración de la imagen social como premisa socio-cultural para la interpretación 
de actividades verbales y no verbales de imagen. En Orletti, F. y Marianotti, L. (Eds.), (Des)cortesía en español. Espacios teóricos y metodológicos para su estudio (pp. 19-47). Roma: Università degli Studi Roma Tre.

3. Bucholtz, M. y Hall, K. (2005). Identity and Interaction: A Sociocultural Linguistic Approach. Discourse Studies, 7(4-5), pp. 585-614. DOI: https://doi.org/10.1177/1461445605054407

4. Byung Chul-Han (2014). En el enjambre. Barcelona. Herder.

5. Byung Chul-Han (2016). Topología de la violencia. Barcelona: Herder.

6. Byung Chul-Han (2018). Hiperculturalidad. Cultura y globalización. Barcelona: Herder.

7. Foucault, M. (1980). Microfisica del poder. Madrid: Las Ediciones de La Piqueta.

8. Foucault, M. (1987). El orden del discurso. Barcelona: Tusquets.

9. Fuentes, C. y Alcaide, E. (2008). (Des)cortesía, agresividad y violencia verbal en la sociedad actual. Sevilla: Universidad Internacional de Andalucía.

10. Goffman, E. (1997). La presentación de la persona en la vida cotidiana. Buenos Aires: Amorrortu Editores.

11. Google Trend (2019). Consulta las tendencias del 2018-México. Recuperado de: https://trends.google.es/trends/yis/2018/MXI.

12. Haidar, J. (2018). Las falacias de la posverdad: desde la complejidad y la transdisciplinariedad. Oxímora. Revista internacional de ética y política, 13, pp. 1-16.

13. Haidar, J. y Rodriguez Alfano, L. (1996). Funcionamiento del poder y de la ideología en las prácticas discursivas. Dimensión Antropológica, 7, pp. 73-111.

14. Hall, S. (1996). ¿Quién necesita identidad? Cuestiones de identidad Cultural, pp. 13-39.

15. Husserl, E. (1986). Meditaciones cartesianas. México: FCE.

16. Lipovetsky, G. (09 de noviembre del 2016a). Espiral-Conversación con Gilles Lipovetsky [video]. Recuperado de:

https://www.youtube.com/watch?v=ZvHyeH6ogNk\&t=3s.

17. Lipovetsky, G. (2016b). La era del vacio. Madrid: Anagrama Océano.

18. Mancera Rueda, A. y Pano Alamán, A. (2013). Nuevas dinámicas discursivas en la comunicación política en Twitter. Círculo de Lingüística Aplicada a la Comunicación, 56, pp. 53-80. DOI: https://doi.org/10.5209/rev_CLAC.2013.v56.43867

19. Pano Alamán, A. (2016). Narrativa colectiva y polifonía en Twitter: del relato colectivo al tuiteo en directo. En Carpi, E., García Jiménez, M. R. y Liverani, E. (Eds.), Le forme del narrare: nel tempo e tra $i$ generi (Vol. 1, pp. 179-197). Trento: Pubblicazioni del Dipartimento di Studi Letterari, Linguistici e Filologici, Università di Trento.

20. Salgado, E. (2018). Del \#SimulacroCDMX al \#MéxicoNosNecesita: 19S en Twitter. Revista Mexicana de Sociología, 80, pp. 21-147.

21. Salgado, E. (2017). Los tuiteros frente al poder: estrategias de la confrontación discursiva. En Salgado, E. y Andrade, L. (Eds.), Estudios del discurso en México. Nuevas prácticas, nuevos enfoques 
(pp. 441- 467). Mérida: UNAM-Centro Peninsular en Humanidades y Ciencias Sociales.

22. Sloterdijk, P. (2003). Crítica de la razón cínica. Madrid: Siruela.

23. Trendinalia (2019). Trending topics. Recuperado el 5 de febrero del 2019 de https://www.trendinalia.com/twitter-trendingtopics/globales/globales-190926.html

24. Van Dijk, T. (2006). Ideología. Una aproximación multidisciplinaria. Barcelona: Gedisa.

25. Winocur, R. y Sánchez J. (2016). Redes sociodigitales en México. Ciudad de México: FCE.

26. Yus, F. (2014). El discurso de las identidades en línea: El caso de Facebook. Discurso y Sociedad, 8(3), pp. 398-426. 